\title{
Anuros da Estação Ecológica de Santa Bárbara, um remanescente de formações abertas de Cerrado no estado de São Paulo
}

\author{
Cybele de Oliveira Araujo ${ }^{1,4}$, Décio Tadeu Corrêa ${ }^{2}$ \& Selma Maria de Almeida Santos ${ }^{3}$ \\ ${ }^{1}$ Seção de Animais Silvestres, Instituto Florestal, Rua do Horto, 931, Horto Florestal, \\ CEP 02377-000, São Paulo, SP, Brasil \\ ${ }^{2}$ Programa de Pós-graduação em Ecologia, Departamento de Biologia Animal, Instituto de Biologia, \\ Universidade Estadual de Campinas - UNICAMP, CP 6109, CEP 13083-862, Campinas, SP, Brasil \\ ${ }^{3}$ Laboratório de Ecologia e Evolução, Instituto Butantan, Avenida Dr. Vital Brasil, 1500, \\ CEP 05503-900, São Paulo, SP, Brasil \\ ${ }^{4}$ Autor para correspondência: Cybele de Oliveira Araujo, e-mail: cyaraujo@if.sp.gov.br
}

ARAUJO, C.O., CORRÊA, D.T. \& ALMEIDA-SANTOS, S.M. Anurans of the Estação Ecológica de Santa Bárbara, a remnant of open Cerrado formations in the state of São Paulo. Biota Neotrop. 13(3): http://www. biotaneotropica.org.br/v13n3/en/abstract?inventory+bn01613032013

\begin{abstract}
Although São Paulo is the state with the most knowledge about anurans from Brazil, it still has important knowledge gaps about this group especially in the remnants of the Cerrado Biome. Only $0.5 \%$ of this biome is protected by conservation units in the state and remaining fragments suffer threats that include isolation, risk of fire and invasion by exotic plant species. The aim of this study was to obtain information on anurans species recorded at Estação Ecológica de Santa Bárbara, one of the few remnants in Middle Paranapanema river basin that has a predominance of open Cerrado formations. The inventory was conducted monthly from October/2008 to March/2009, totaling 30 sampling days. Surveys were conducted using pitfall traps with drift fences, incidental encounters and acoustic and visual searches. We recorded 33 anuran species, belonging to 14 genera and six families (Bufonidae, Cycloramphidae, Hylidae, Leiuperidae, Leptodactylidae e Microhylidae). Although species richness was not different between open and forest vegetation types, there was a greater abundance of anurans in the open formations, reflecting the largest association of individuals to water bodies present in open phytophysiognomies during the reproductive period. This research is an important contribution to expanding the knowledge about anurans species in the Cerrado, one of the priority global hotspots for biodiversity conservation.
\end{abstract}

Keywords: amphibians, Anura, conservation, inventory, Águas de Santa Bárbara.

ARAUJO, C.O., CORRÊA, D.T. \& ALMEIDA-SANTOS, S.M. Anuros da Estação Ecológica de Santa Bárbara, um remanescente de formações abertas de Cerrado no estado de São Paulo. Biota Neotrop. 13(3): http:// www.biotaneotropica.org.br/v13n3/pt/abstract?inventory+bn01613032013

Resumo: Apesar de São Paulo ser o estado do país que possui o maior conhecimento sobre sua fauna de anuros, ainda persistem consideráveis vazios amostrais, sobretudo nos remanescentes de Cerrado do estado. Somente $0,5 \%$ deste bioma encontram-se protegidos por unidades de conservação no estado e os fragmentos remanescentes sofrem ameaças que incluem o isolamento, risco de incêndios e invasão por espécies vegetais exóticas. Neste contexto, o objetivo deste estudo foi gerar informações sobre as espécies de anuros presentes na Estação Ecológica de Santa Bárbara, um dos poucos remanescentes com formações abertas de Cerrado da bacia hidrográfica do Médio Paranapanema. O levantamento das espécies foi realizado mensalmente entre outubro/2008 a março/2009, totalizando 30 dias de inventário. A amostragem foi realizada por meio de armadilhas de interceptação e queda, encontro ocasional, procura auditiva e procura visual. Registramos 33 espécies pertencentes a 14 gêneros e seis famílias (Bufonidae, Cycloramphidae, Hylidae, Leiuperidae, Leptodactylidae e Microhylidae). Embora a riqueza de espécies não tenha sido diferente entre as formações vegetais abertas e florestais, registrou-se uma maior abundância de anuros nas formações savânicas, refletindo a maior associação dos indivíduos aos corpos d'água presentes nas fitofisionomias abertas durante o período reprodutivo. Este estudo é uma importante contribuição para ampliar o conhecimento sobre a fauna de anuros no Cerrado, um dos hotspots mundiais prioritários para a conservação biológica.

Palavras-chave: anfíbios, Anura, conservação, inventário, Águas de Santa Bárbara. 


\section{Introdução}

Apesar da fauna de anuros do estado de São Paulo ser considerada a mais conhecida no país, com 230 espécies descritas, ainda existem consideráveis lacunas amostrais no nordeste e sudeste do estado (Rossa-Feres et al. 2011). Embora tenha ocorrido nos últimos anos um aumento significativo no número de trabalhos publicados sobre a diversidade de anuros em localidades da bacia hidrográfica do Médio Paranapanema (Ribeiro-Júnior \& Bertoluci 2009, Araujo \& AlmeidaSantos 2011, Maffei et al. 2011a, b), o conhecimento sobre este grupo faunístico nesta região continua restrito quando comparado a outras regiões do estado (Rossa-Feres et al. 2011). A anurofauna do estado pode ser dividida entre as espécies distribuídas nas fitofisionomias de floresta ombrófila da encosta litorânea e serras do Mar, Mantiqueira e Bocaina e as associadas às formações de Cerrado e floresta estacional que ocorrem predominantemente no Planalto Ocidental Paulista (Rossa-Feres et al. 2008).

O Cerrado é o segundo maior domínio neotropical e o único dos 34 hotspots mundiais prioritários para a conservação biológica em que predomina a vegetação savânica (Myers et al. 2000, Mittermeier et al. 2004). A vegetação que possuía originalmente dois milhões de $\mathrm{km}^{2}$ de extensão ( $23 \%$ do território nacional) compreende uma extensa área continua nos estados de Goiás, Bahia, Minas Gerais e Mato Grosso e algumas penínsulas e áreas disjuntas que se estendem por outros estados (Eiten 1972). Sua heterogeneidade ambiental, que inclui planaltos, depressões e planícies dominadas por formações campestres e savânicas e em menor proporção matas de galeria e floresta estacional (Oliveira-Filho \& Ratter 2002, Silva \& Bates 2002, Ab'Sáber 2005, Ribeiro \& Walter 2008), está refletida em sua biota reconhecida mundialmente como uma das mais ricas em espécies e endemismos e severamente ameaçada pelas atividades humanas (Colli et al. 2002, Mittermeier et al. 2004, Klink \& Machado 2005, Diniz-Filho et al. 2008). Estudo recente aponta uma elevada riqueza de espécies e endemismos de anfíbios no Cerrado, com 209 espécies de anuros, sendo 51,7\% endêmicas deste domínio (Valdujo et al. 2012). Aproximadamente $40 \%$ da cobertura vegetal do Cerrado foram devastadas e a porção mais ao sul deste domínio é a mais severamente impactada, apresentando apenas $15 \%$ de sua vegetação original (Sano et al. 2010). É justamente nas regiões sul e sudoeste deste domínio, que a riqueza de espécies de anuros tende a ser mais elevada (Diniz-Filho et al. 2008, Valdujo 2011).

As características ecológicas, fisiológicas e reprodutivas dos anuros levam este grupo a uma forte associação aos ambientes úmidos (Duellman \& Trueb 1986, Pough et al. 2004). Em regiões de Cerrado, a maior parte destas espécies ocorre em ambientes abertos e úmidos, como nas fitofisionomias campo limpo úmido e veredas, e os endemismos concentram-se nos campos limpos e campos rupestres (Brandão \& Araújo 2002, Colli et al. 2002, Eterovick \& Sazima 2004, Brasileiro et al. 2005, Araujo et al. 2009, Valdujo 2011, Valdujo et al. 2011). É importante destacar que as fisionomias campestres e savânicas são as formações vegetais mais vulneráveis e sujeitas ao desaparecimento no estado de São Paulo (Durigan et al. 2003, Durigan et al. 2004, Durigan \& Ratter 2006, Durigan et al. 2007), restando poucas unidades de conservação de proteção integral que ainda abrigam áreas significativas destas fitofisionomias no estado como as estações ecológicas de Itirapina e Santa Bárbara (Kronka et al. 2005). Análises da cobertura vegetal em fragmentos considerados como prioritários para a conservação do Cerrado no estado demonstraram que as formações savânicas ocorrem em $35 \%$ dos fragmentos e as formações campestres em apenas 3,5\%, sendo o restante dominado pelas formações florestais (Durigan et al. 2003). Somente $0,5 \%$ deste domínio encontra-se protegido por unidades de conservação no estado e os fragmentos remanescentes sofrem ameaças que incluem o isolamento, risco de incêndios e invasão por espécies vegetais exóticas (Pivello et al. 1999, Durigan et al. 2004, Durigan et al. 2007).
Considerando que estudos realizados no Cerrado prevêem, em um cenário de mudanças climáticas, alterações na disponibilidade de áreas adequadas para ocorrência de muitas espécies de anuros, em especial as endêmicas e especialistas no uso do hábitat (Silvano 2011), torna-se imperativo a realização de inventários e estudos padronizados de monitoramento dos anuros em localidades que se mantêm como lacunas amostrais neste domínio fitogeográfico. Os dados obtidos neste tipo de pesquisa podem contribuir para uma avaliação mais adequada do status de conservação das espécies, declínios populacionais e extinções locais, informações estas indispensáveis para a elaboração de medidas efetivas de manejo e conservação das espécies (Verdade et al. 2012). Possivelmente, as populações de anuros registradas na Estação Ecológica de Santa Bárbara representam o limite de distribuição mais austral destas espécies neste domínio (Diniz-Filho et al. 2008, Valdujo 2011). Antes deste estudo, só eram conhecidas três espécies para o município de Águas de Santa Bárbara (C. Nogueira, dados não publicados). Neste contexto, o objetivo deste trabalho foi fornecer informações sobre a composição, riqueza, abundância e distribuição dos anuros nesta unidade de conservação. Adicionalmente, considerando que vários estudos apontaram que as formações abertas abrigam uma maior diversidade de anuros em relação às formações florestais de Cerrado (Brandão \& Araújo 2002, Brasileiro et al. 2005, Araujo et al. 2009, Valdujo 2011, Valdujo et al. 2011), testou-se nesta localidade a proposição que os ambientes com fisionomias abertas apresentam uma maior riqueza e abundância de espécies quando comparados às fisionomias florestais.

\section{Material e Métodos}

\section{Local de estudo}

A Estação Ecológica de Santa Bárbara (22 46'-22 $41^{\circ}$ ' S, $49^{\circ} 16^{\prime}$ $49^{\circ} 10^{\prime} \mathrm{O}$; 600-680 m) localiza-se no município de Águas de Santa Bárbara (Médio Paranapanema), estado de São Paulo e apresenta área total de 2.712 ha (Melo \& Durigan 2011) (Figura 1). O clima da região é do tipo Cwa de Köppen, com uma estação seca e outra chuvosa (Peel et al. 2007). A temperatura média nos meses mais quentes (janeiro e fevereiro) oscila entre 24 e $24,5^{\circ} \mathrm{C}$ e nos meses mais frios (junho e julho) ao redor de $17^{\circ} \mathrm{C}$ (Melo \& Durigan 2011). A precipitação média anual é de aproximadamente $1.400 \mathrm{~mm}$, com a maior média de precipitação mensal ocorrendo no mês de dezembro (206 mm) e a menor em agosto (44 mm) (Melo \& Durigan 2011). A cobertura vegetal predominante é de Cerrado, sendo composta sobretudo por formações campestres e savânicas, como cerrado típico $(34,4 \%)$, cerrado denso $(10,2 \%)$, cerrado ralo (7\%) e campo limpo úmido (6,5\%) (Durigan et al. 2011). As fisionomias florestais naturais são o cerradão $(11,9 \%)$, mata de galeria (4\%) e mata seca semidecídua (0,7\%) (Durigan et al. 2011). As fitofisionomias foram classificadas sensu Ribeiro e Walter (2008), na qual o cerrado sentido restrito é subdividido em cerrado denso, cerrado típico e cerrado ralo.

\section{Coleta e análise dos dados}

O inventário das espécies foi realizado em seis etapas de campo mensais, com duração de cinco dias cada, totalizando 30 dias de amostragem (outubro/2008 a março/2009). Foram utilizadas armadilhas de interceptação e queda (pitfall traps) interligadas por cercas-guia (drift-fences) (Corn 1994) em um ambiente com formações florestais (cerradão e mata seca semidecídua) e em dois ambientes com formações savânicas (cerrado típico e cerrado ralo) (Figura 2, Apêndice 1). Adicionalmente, foram realizadas amostragens por meio dos métodos de procura auditiva, procura visual e encontro ocasional (Crump \& Scott Junior 1994, Scott Junior \& Woodward 1994) nos ambientes descritos acima e nos sítios reprodutivos, especialmente em brejos formados no campo limpo 
úmido (Figura 2, Apêndice 1). Também foram considerados alguns registros de espécies obtidos em inventário realizado após o término deste estudo (2011/2012).

Foram instaladas 18 linhas de armadilhas (unidades amostrais) compostas por quatro baldes plásticos de 60 litros, totalizando

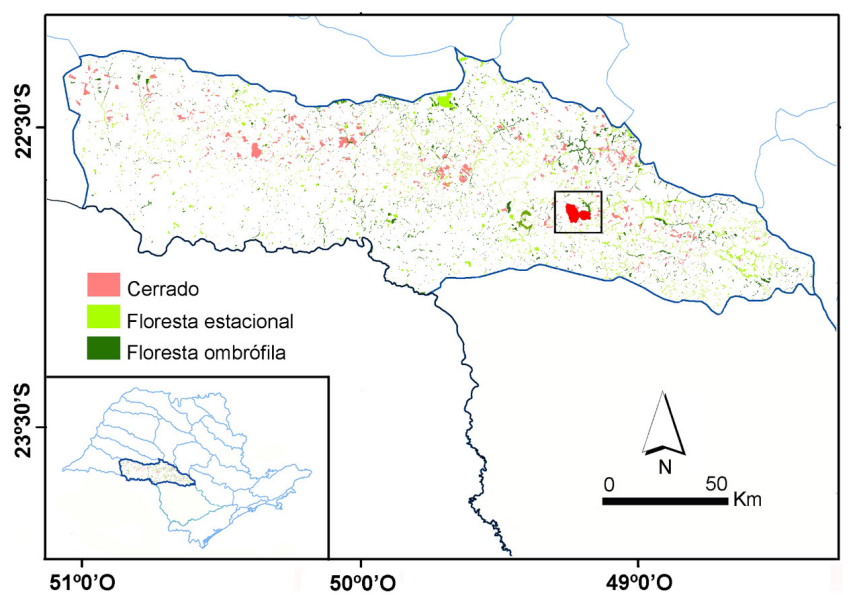

Figura 1. Remanescentes de vegetação natural da bacia hidrográfica do Médio Paranapanema, SP. O fragmento em vermelho representa a Estação Ecológica de Santa Bárbara. No detalhe à esquerda, estão representadas as bacias hidrográficas do estado de São Paulo, com destaque para o Médio Paranapanema.

Figure 1. Natural vegetation remnants of the Middle Paranapanema river basin, São Paulo state, Brazil. The fragment in focus (in red) represents the Estação Ecológica de Santa Bárbara. The detail on the left side depicts the river basins of São Paulo state, highlighting the Middle Paranapanema.
72 armadilhas, com uma distância mínima de $500 \mathrm{~m}$ entre linhas (Figura 2, Apêndice 1). Os baldes foram inseridos em buracos cavados no solo, sendo dispostos em linha reta (espaçados 15 metros entre si) e interligados por cercas-guia de aproximadamente $50 \mathrm{~cm}$ de altura. As armadilhas permaneceram abertas por cinco dias consecutivos (primeira ou segunda semana do mês), em um total de 30 dias de amostragem (esforço amostral $=2.160$ armadilhas/dia). Com o intuito de minimizar a predação dos anuros capturados nas armadilhas por vertebrados e invertebrados foram realizadas inspeções a cada 24 horas, sempre no período da manhã. Foram depositados abrigos em cada uma das armadilhas durante todo o período de amostragem para evitar a morte de indivíduos por dessecação e afogamento (Cechin \& Martins 2000). Foram coletados no máximo 10 indivíduos por espécie. Os demais espécimes capturados foram identificados, marcados pela amputação da falange distal do terceiro artelho do membro anterior direito (toe-tipping) (Lüddecke \& Amézquita 1999, Phillott et al. 2007) e liberados próximos ao local de captura (licenças IBAMA/RAN 10423-1 e 13706-2). Este tipo de marcação (batch mark) foi utilizado para que os indivíduos recapturados nas armadilhas não fossem recontados nas amostragens subsequentes. A relevância deste método de marcação de anuros em estudos populacionais foi apresentada recentemente por Corrêa et al. (2013).

A procura auditiva e visual consistiu em censos efetuados no período noturno, sendo examinados os microhábitats acessíveis (e.g. bromélias, folhiço, tronco de árvores) e os sítios reprodutivos utilizados pelas espécies (e.g. brejos, córregos, lagoas, várzeas). A amostragem variou entre três e seis horas por noite, resultando em um esforço total de aproximadamente 120 horas de procura e esforço empregado de 60 horas-homem. Grande parte dos ambientes aquáticos utilizados pelos anuros durante a reprodução nesta localidade foi inspecionado ao longo dos 14 dias de amostragem ativa (Figura 2, Apêndice 1). O número de sítios aquáticos vistoriados e a

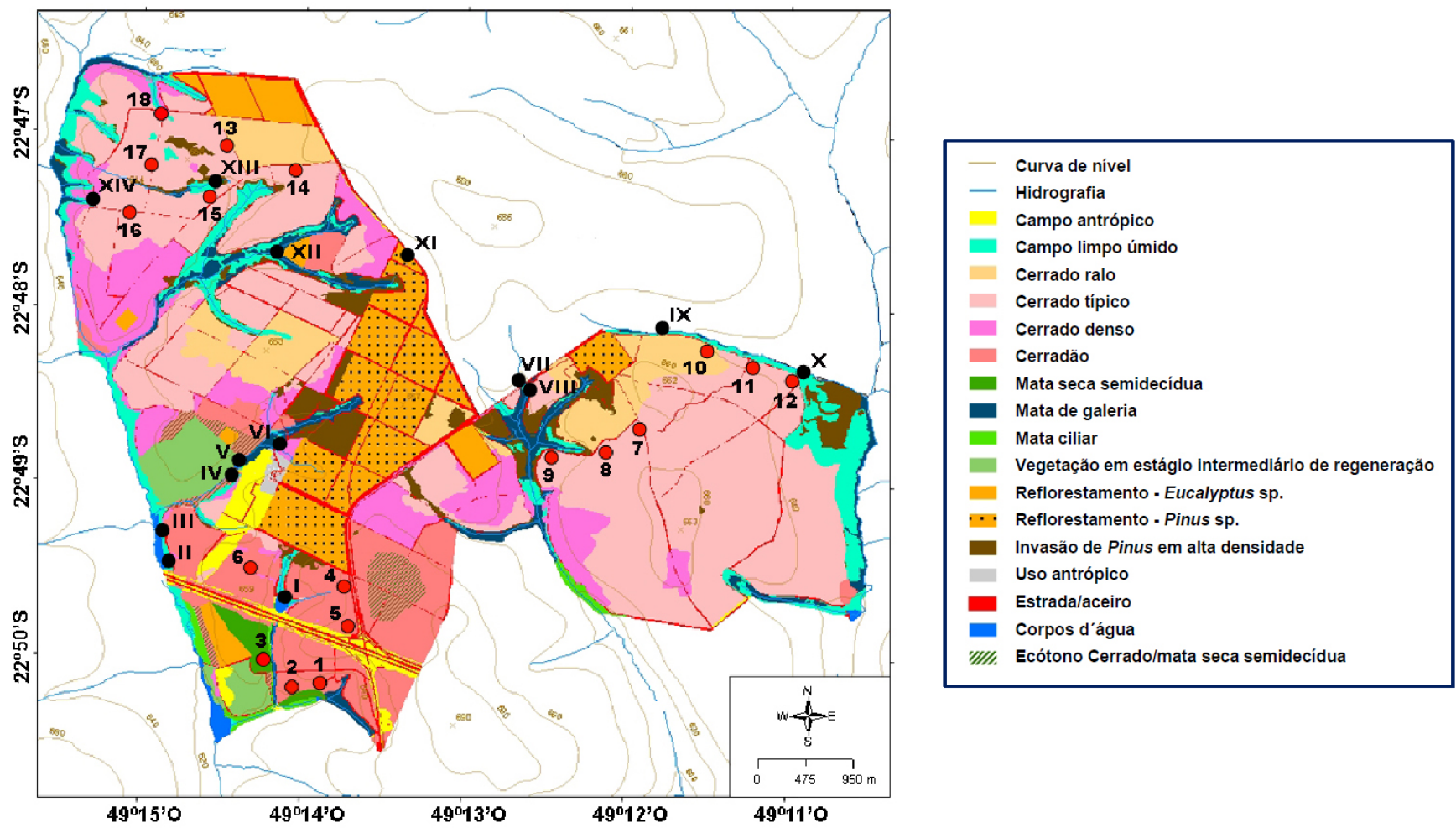

Figura 2. Cobertura vegetal da Estação Ecológica de Santa Bárbara, SP e locais de amostragem (pontos em vermelho = linhas de armadilhas de interceptação e queda; pontos em preto = ambientes aquáticos). Fonte: modificado de Melo \& Durigan (2011).

Figure 2. Vegetation of the Estação Ecológica de Santa Bárbara, São Paulo state, Brazil and sampled areas (red dots = pitfall trap lines; black dots = aquatic sites). Source: modified from Melo \& Durigan (2011). 
distância percorrida em cada noite não foram padronizados, no entanto manteve-se o compromisso de amostrar todos os sítios reprodutivos em cada etapa mensal. O método de encontro ocasional consistiu no registro de espécimes encontrados vivos ou mortos, quando não estava sendo executado nenhum tipo de procura. Os espécimes testemunho foram depositados na Coleção de Anfíbios do Instituto de Biociências da Universidade Estadual Paulista, Rio Claro (CFBH) e Coleção Herpetológica do Museu de Zoologia da Universidade de São Paulo (MZUSP) (Apêndice 2).

A eficiência da amostragem foi avaliada por meio de curvas de rarefação (Gotelli \& Colwell 2001) geradas a partir dos dados de incidência dos anuros durante o período de inventário. O número total de espécies registradas em cada dia foi considerado como uma amostra, totalizando 14 amostras obtidas pela procura auditiva e visual e 30 amostras pelas linhas de armadilhas. Considerando os diversos estimadores de riqueza disponíveis, optou-se pela utilização do algoritmo Jackknife de primeira ordem (Magurran 2004, Walther \& Moore 2005, Hortal et al. 2006). Apesar de alguns autores apontarem que os estimadores não-paramétricos apresentam uma grande dependência da riqueza de espécies observada e, portanto, do número e tamanho das amostras (Melo \& Froehlich 2001, Melo 2004), ainda assim a riqueza estimada neste inventário pode fornecer uma base para comparações com outros levantamentos realizados em localidades do interior do estado que utilizaram este estimador de riqueza (e.g. Ribeiro-Júnior \& Bertoluci 2009, Santos et al. 2009, Brassaloti et al. 2010, Araujo \& Almeida-Santos 2011, Valdujo et al. 2011, Araujo \& Almeida-Santos 2013). Para averiguar se existem diferenças na riqueza de espécies entre os ambientes abertos e florestais foram avaliados apenas os dados obtidos pelas armadilhas. Para tal, curvas de rarefação foram construídas baseadas no número de indivíduos registrados e comparou-se a riqueza de espécies considerando o ambiente que apresentou o menor número de anuros capturados, que no caso foi o ambiente florestal (Gotelli \& Colwell 2001). Todas as análises foram realizadas no programa EstimateS v.8.2.0 com 10.000 aleatorizações (Colwell 2009). Devido à dificuldade na diferenciação taxonômica dos juvenis, as espécies Physalaemus centralis e $P$. cuvieri foram consideradas conjuntamente para a determinação da riqueza de espécies e contagem de indivíduos.

Apesar da existência de um sistema de classificação mais recente para os anuros (Pyron \& Wiens 2011), optou-se pela utilização da nomenclatura taxonômica mais consagrada apresentada por Frost (2013). O status de conservação das espécies foi obtido na lista vermelha internacional de espécies ameaçadas de extinção (International... 2013), livro vermelho da fauna brasileira ameaçada de extinção (Machado et al. 2008) e lista de anfíbios ameaçados do estado de São Paulo (Garcia et al. 2009).

\section{Resultados e Discussão}

\section{Composição e distribuição das espécies}

Registraram-se na Estação Ecológica de Santa Bárbara 33 espécies de anuros distribuídas em seis famílias: Bufonidae (2 espécies), Cycloramphidae (1 espécie), Hylidae (15 espécies), Leiuperidae (5 espécies), Leptodactylidae (7 espécies) e Microhylidae (3 espécies) (Figura 3, Tabela 1). Deste total, 30 espécies representam novos registros para o município de Águas de Santa Bárbara, duas espécies já haviam sido observadas nesta localidade (Leptodactylus mystacinus $\mathrm{e}$ Elachistocleis cesarii) e apenas a espécie Odontophrynus cf. cultripes foi encontrada exclusivamente na Coleção Herpetológica do Museu de Zoologia da Universidade de São Paulo (MZUSP) (Tabela 1). Contudo, o registro de $O$. cf. cultripes deve ser visto com cautela, pois apesar de constar no livro de registros da referida coleção, o indivíduo não foi localizado no acervo. A captura de Pseudopaludicola $\mathrm{cf}$. murundu neste estudo representou o primeiro registro da espécie para a bacia hidrográfica do Médio Paranapanema. Nenhuma das espécies estudadas encontra-se nas listas de anfíbios ameaçados de extinção (International... 2013, Machado et al. 2008, Garcia et al. 2009). Entretando, as populações de anuros desta localidade podem representar o limite de distribuição mais austral das espécies neste domínio fitogeográfico (Diniz-Filho et al. 2008, Valdujo 2011) e a conservação e conexão destes remanescentes de Cerrado contribuem para a manutenção da diversidade genética destas espécies.

Parte das espécies (27\%) apresentam uma ampla distribuição geográfica, sendo comumente encontradas em várias regiões do Brasil como Dendropsophus minutus, D. nanus, Hypsiboas albopunctatus, Leptodactylus fuscus, L. mystacinus, Physalaemus cuvieri, Rhinella schneideri, Scinax fuscomarginatus e $S$. fuscovarius (Haddad et al. 2008, Frost 2013). No entanto, uma parcela significativa (30\%) está fortemente associada ao domínio do Cerrado como Hypsiboas lundii, típica das formações florestais e Chiasmocleis albopunctata, Dendropsophus elianeae, D. jimi, Eupemphix nattereri, Leptodactylus furnarius, L. jolyi, Odontophrynus cf. cultripes, Physalaemus centralis e $P$. marmoratus que apresentam uma grande dependência das formações vegetais abertas presentes neste domínio (Toledo et al. 2007, Uetanabaro et al. 2008, Valdujo 2011).

Das espécies registradas, Leptodactylus mystaceus e Rhinella ornata não foram avistadas em atividade de vocalização (Tabela 1). Grande parte dos anuros (18 espécies; 54\%) foi observada em atividade reprodutiva restrita aos ambientes aquáticos presentes nas formações abertas, sendo que somente Aplastodiscus perviridis, Hypsiboas lundii, Itapotihyla langsdorffii e Phyllomedusa tetraploidea foram encontradas vocalizando exclusivamente nos ambientes aquáticos florestais (Tabela 1, Apêndice 1). A maior associação das espécies aos corpos d'água presentes nas formações abertas durante o período reprodutivo já foi demonstrada em outros estudos que compararam a riqueza de espécies em formações campestres, savânicas e florestais em regiões de Cerrado (e.g. Brandão \& Araújo 2002, Brasileiro et al. 2005, Araujo et al. 2009, Valdujo et al. 2011). Apenas Hypsiboas lundii foi observada vocalizando em corpos d'água lóticos (e.g. riachos), sendo a maior parte das espécies $(79 \%)$ encontrada em atividade de vocalização unicamente em corpos d'água lênticos (e.g. brejos, charcos, lagoas). Somente Aplastodiscus perviridis, Hypsiboas albopunctatus, H. caingua, Itapotihyla langsdorffii e Physalaemus cuvieri foram encontradas em ambos os tipos de ambiente aquático (17\%) (Tabela 1, Apêndice 1). Padrão semelhante no uso do hábitat para a reprodução foi verificado em outras localidades que apresentam formações de Cerrado (e.g. Brasileiro et al. 2005, Araujo et al. 2009, Araujo \& Almeida-Santos 2011, Valdujo et al. 2011).

\section{Riqueza e abundância das espécies}

A riqueza de espécies observada na Estação Ecológica de Santa Bárbara mostrou-se superior às registradas em outras unidades de conservação que apresentam predomínio de formações de Cerrado no estado de São Paulo como as estações ecológicas de Assis (27 espécies; Araujo \& Almeida-Santos 2011) e Itirapina (28 espécies; Brasileiro et al. 2005) e áreas de proteção integral no interior do estado que apresentam ecótono entre o Cerrado e a Mata Atlântica como as estações ecológicas de Angatuba (31 espécies; Araujo \& AlmeidaSantos 2013) e Jataí (27 espécies; D. T. Corrêa, comunicação pessoal).

As curvas de rarefação para 14 dias de amostragem por procura auditiva e visual e 30 dias por armadilhas de interceptação e queda não atingiram uma assíntota. Contudo, no caso da procura ativa, as curvas apresentaram tendência à estabilização, indicando que possivelmente a maior parte das espécies foi registrada durante o estudo (Figura 4). O estimador de riqueza Jackknife I não indicou diferenças entre as riquezas de anuros estimadas e observadas obtidas pelos diferentes métodos de amostragem (Figura 4). Grande parte das espécies (29 espécies, 90\%), incluindo a observação de Itapotihyla langsdorffii após o término deste estudo, foi amostrada por meio da procura auditiva e visual nos ambientes aquáticos utilizados 

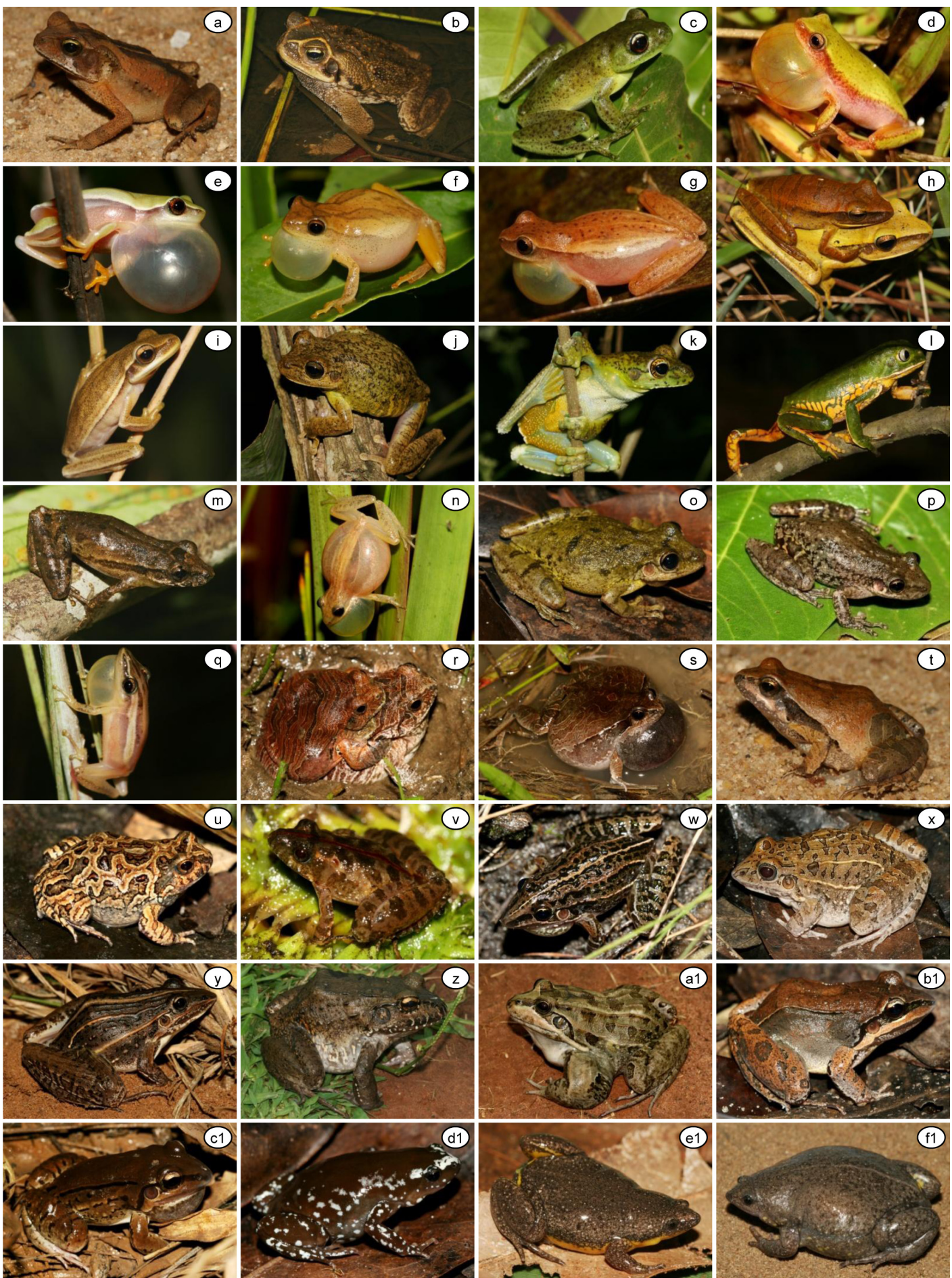

Figura 3. Espécies de anuros amostradas na Estação Ecológica de Santa Bárbara, SP. $\mathrm{a}=$ Rhinella ornata $; \mathrm{b}=$ Rhinella schneideri; $\mathrm{c}=$ Aplastodiscus perviridis; $\mathrm{d}=$ Dendropsophus elianeae $; \mathrm{e}=$ Dendropsophus jimi $; \mathrm{f}=$ Dendropsophus minutus $; \mathrm{g}=$ Dendropsophus nanus $; \mathrm{h}=$ Hypsiboas albopunctatus $; \mathrm{i}=$ Hypsiboas caingua $; \mathrm{j}=$ Hypsiboas lundii $; \mathrm{k}=$ Itapotihyla langsdorffii $; \mathrm{l}=$ Phyllomedusa tetraploidea $; \mathrm{m}=$ Scinax berthae $; \mathrm{n}=$ Scinax fuscomarginatus $; \mathrm{o}=$ Scinax fuscovarius; $\mathrm{p}=$ Scinax similis; $\mathrm{q}=$ Scinax squalirostris $; \mathrm{r}=$ Eupemphix nattereri; $\mathrm{s}=$ Physalaemus centralis; $\mathrm{t}=$ Physalaemus cuvieri; $\mathrm{u}=$ Physalaemus marmoratus $; \mathrm{v}=$ Pseudopaludicola $\mathrm{cf}$. murundu; $\mathrm{w}=$ Leptodactylus furnarius $; \mathrm{x}=$ Leptodactylus fuscus $; \mathrm{y}=$ Leptodactylus jolyi $; \mathrm{z}=$ Leptodactylus labyrinthicus; $\mathrm{a} 1$ = Leptodactylus latrans; $\mathrm{b} 1$ = Leptodactylus mystaceus; $\mathrm{c} 1=$ Leptodactylus mystacinus; $\mathrm{d} 1=$ Chiasmocleis albopunctata $; \mathrm{e} 1=$ Elachistocleis bicolor; $\mathrm{f} 1$ = Elachistocleis cesarii. Todos os indivíduos são da localidade estudada (fotos: Cybele O. Araujo).

Figure 3. Anuran species sampled at the Estação Ecológica de Santa Bárbara, São Paulo state, Brazil. $\mathrm{a}=$ Rhinella ornata; $\mathrm{b}=$ Rhinella schneideri; $\mathrm{c}=$ Aplastodiscus perviridis $; \mathrm{d}=$ Dendropsophus elianeae $; \mathrm{e}=$ Dendropsophus jimi $; \mathrm{f}=$ Dendropsophus minutus $; \mathrm{g}=$ Dendropsophus nanus $; \mathrm{h}=$ Hypsiboas albopunctatus $; \mathrm{i}=$ Hypsiboas caingua $; \mathrm{j}=$ Hypsiboas lundii $; \mathrm{k}=$ Itapotihyla langsdorffi $; \mathrm{l}=$ Phyllomedusa tetraploidea $; \mathrm{m}=$ Scinax berthae $; \mathrm{n}=$ Scinax fuscomarginatus $; \mathrm{o}=$ Scinax fuscovarius $; \mathrm{p}=$ Scinax similis $; \mathrm{q}=$ Scinax squalirostris; $\mathrm{r}=$ Eupemphix nattereri $; \mathrm{s}=$ Physalaemus centralis; $\mathrm{t}=$ Physalaemus cuvieri $; \mathrm{u}=$ Physalaemus marmoratus $; \mathrm{v}=$ Pseudopaludicola $\mathrm{cf}$. murundu; $\mathrm{w}=$ Leptodactylus furnarius $; \mathrm{x}=$ Leptodactylus fuscus; $\mathrm{y}=$ Leptodactylus jolyi; $\mathrm{z}=$ Leptodactylus labyrinthicus; $\mathrm{a} 1$ = Leptodactylus latrans; $\mathrm{b} 1=$ Leptodactylus mystaceus; $\mathrm{c} 1=$ Leptodactylus mystacinus; $\mathrm{d} 1=$ Chiasmocleis albopunctata; e1 = Elachistocleis bicolor; $\mathrm{fl}=$ Elachistocleis cesarii. All individuals were found at the study site (photos: Cybele O. Araujo). 
Anuros da Estação Ecológica de Santa Bárbara, estado de São Paulo

Tabela 1. Anuros da Estação Ecológica de Santa Bárbara, SP. Métodos de amostragem: AIQ = armadilhas de interceptação e queda; PA = procura auditiva; $\mathrm{PV}=$ procura visual. Locais de captura: linhas de armadilhas e ambientes aquáticos.

Table 1. Anurans from Estação Ecológica de Santa Bárbara, São Paulo state, Brazil. Sampling methods: AIQ = pitfall traps; PA= auditory encounters; PV = visual encounters. Capture sites: pitfall traps lines and aquatic sites.

\begin{tabular}{|c|c|c|}
\hline Família/espécie & Método & Linha de armadilha/ambiente aquático \\
\hline \multicolumn{3}{|l|}{ Bufonidae } \\
\hline Rhinella ornata (Spix 1824) & AIQ & $1,2,3$ \\
\hline Rhinella schneideri (Werner 1894) & $\mathrm{PA}, \mathrm{PV}$ & I, VI, XII \\
\hline \multicolumn{3}{|l|}{ Cycloramphidae } \\
\hline Odontophrynus cf. cultripes (Reinhardt \& Lütken 1862) & - & - \\
\hline \multicolumn{3}{|l|}{ Hylidae } \\
\hline Aplastodiscus perviridis A. Lutz in B. Lutz 1950 & PA, PV & VIII \\
\hline Dendropsophus elianeae (Napoli \& Caramaschi 2000) & $\mathrm{PA}, \mathrm{PV}$ & VI \\
\hline Dendropsophus jimi (Napoli \& Caramaschi 1999) & $\mathrm{PA}, \mathrm{PV}$ & I, II, III, IX, X, XIII \\
\hline Dendropsophus minutus (Peters 1872) & PA, PV & I, II, III, V, VI, IX, X, XIII \\
\hline Dendropsophus nanus (Boulenger 1889) & PA, PV & I, II, III, V, VI, VII, XI \\
\hline Hypsiboas albopunctatus (Spix 1824) & $\mathrm{PA}, \mathrm{PV}$ & I, II, III, IV, V, VI, VII, VIII, IX, X, XIV \\
\hline Hypsiboas caingua (Carrizo 1991 “1990”) & PA, PV & II, III, V, VII, VIII, IX, X \\
\hline Hypsiboas lundii (Burmeister 1856) & PA, PV & IV, VIII, XII \\
\hline Itapotihyla langsdorffii (Duméril \& Bibron 1841) & $\mathrm{PA}, \mathrm{PV}$ & VIII \\
\hline Phyllomedusa tetraploidea Pombal \& Haddad 1992 & PA, PV & XI \\
\hline Scinax berthae (Barrio 1962) & $\mathrm{PA}, \mathrm{PV}$ & IX, VII \\
\hline Scinax fuscomarginatus (A. Lutz 1925) & $\mathrm{PA}, \mathrm{PV}$ & I, II, III, V, VI, IX, X, XIII \\
\hline Scinax fuscovarius (A. Lutz 1925) & PA, PV & I, VI \\
\hline Scinax similis (Cochran 1952) & PA, PV & VI, IX, X, XIII \\
\hline Scinax squalirostris (A. Lutz 1925) & PA, PV & X, XIII, XIV \\
\hline \multicolumn{3}{|l|}{ Leiuperidae } \\
\hline Eupemphix nattereri Steindachner 1863 & AIQ, PA, PV & $5,7,10,11,12,14,16,17,18, \mathrm{~V}, \mathrm{VI}$ \\
\hline Physalaemus centralis Bokermann 1962 & AIQ, PA, PV & 6, I, II, VI, XIII \\
\hline Physalaemus cuvieri Fitzinger 1826 & AIQ, PA, PV & $\begin{array}{c}\text { 1, } 2,3,6,7,8,9,10,11,12,13,14,15,16,17,18 \text {, I, } \\
\text { II, III, IV, V, VI, VII, VIII, IX, X, XIII, XIV }\end{array}$ \\
\hline Physalaemus marmoratus (Reinhardt \& Lütken 1862 “1861”) & AIQ, PA, PV & $13,14,15,16,17,18, \mathrm{VI}$ \\
\hline $\begin{array}{l}\text { Pseudopaludicola } \text { cf. murundu Toledo, Siqueira, Duarte, } \\
\text { Veiga-Menoncello, Recco-Pimentel \& Haddad } 2010\end{array}$ & PA, PV & $\mathrm{X}$ \\
\hline \multicolumn{3}{|l|}{ Leptodactylidae } \\
\hline Leptodactylus furnarius Sazima \& Bokermann 1978 & AIQ, PA, PV & $13, \mathrm{X}, \mathrm{XIV}$ \\
\hline Leptodactylus fuscus (Schneider 1799) & AIQ, PA, PV & 13, 14, 15, V, VI, VII, VIII, IX, X, XIII \\
\hline Leptodactylus jolyi Sazima \& Bokermann 1978 & AIQ, PA, PV & $17, \mathrm{X}$ \\
\hline Leptodactylus labyrinthicus (Spix 1824) & PA, PV & X, XIII, XIV \\
\hline Leptodactylus latrans (Steffen 1815) & AIQ, PA, PV & 17, VI, X, XIII \\
\hline Leptodactylus mystaceus (Spix 1824) & AIQ & 3 \\
\hline Leptodactylus mystacinus (Burmeister 1861) & AIQ, PA, PV & $6,11,13,14,15,16,17,18, \mathrm{~V}, \mathrm{X}, \mathrm{XI}, \mathrm{XIII}$ \\
\hline \multicolumn{3}{|l|}{ Microhylidae } \\
\hline Chiasmocleis albopunctata (Boettger 1885) & AIQ, PA, PV & $7,9,13,15,18$, II, XIII \\
\hline Elachistocleis bicolor (Valenciennes in Guérin-Menéville 1838) & AIQ & 15 \\
\hline Elachistocleis cesarii (Miranda-Ribeiro 1920) & AIQ, PA, PV & $\begin{array}{c}8,10,11,14,15,16,18, \text { II, VI, VII, VIII, IX, X, } \\
\text { XIII, XIV }\end{array}$ \\
\hline
\end{tabular}

pelos anuros durante o período reprodutivo, sendo que as espécies da família Hylidae (15 espécies) foram observadas exclusivamente por estes métodos (Tabela 1). Nas armadilhas de interceptação e queda, considerando o registro posterior de Elachistocleis bicolor, foram capturadas 14 espécies (44\%) (Tabela 1). As armadilhas de interceptação e queda são mais apropriadas para o registro de espécies de hábitos terrestres como anuros das famílias Bufonidae, Cycloramphidae, Leiuperidae, Leptodactylidae e Microhylidae, especialmente as espécies subterrâneas e criptozóicas, que são mais difíceis de serem detectadas pelos métodos tradicionais de procura visual (Cechin \& Martins 2000). Considerando os anuros inventariados pertencentes a estas famílias, apenas três espécies não foram capturadas por este método (Leptodactylus labyrinthicus, Pseudopaludicola cf. murundu e Rhinella schneideri) (Tabela 1).

É importante destacar que existem limitações inerentes a cada método e apenas a utilização de métodos complementares (amostragem ativa e passiva) propicia a obtenção de listas de espécies mais completas (Silva 2010, Silveira et al. 2010). Embora os custos financeiros e o esforço empregado na instalação e manutenção das armadilhas de interceptação e queda sejam mais elevados que os 
necessários para a realização da procura auditiva e visual (Corn 1994, Cechin \& Martins 2000) é desejável que a amostragem passiva seja empregada em inventários da herpetofauna. Isto porque, além de permitir a amostragem simultânea de diversos locais, este método permite a obtenção de dados padronizados de riqueza e abundância das espécies, o que favorece comparações mais confiáveis entre diferentes localidades (Silveira et al. 2010).

Entre outubro de 2008 a março de 2009, foram capturados nas armadilhas 352 indivíduos de anuros pertencentes a 12 espécies (Figura 5). No ambiente com formações florestais foram registradas
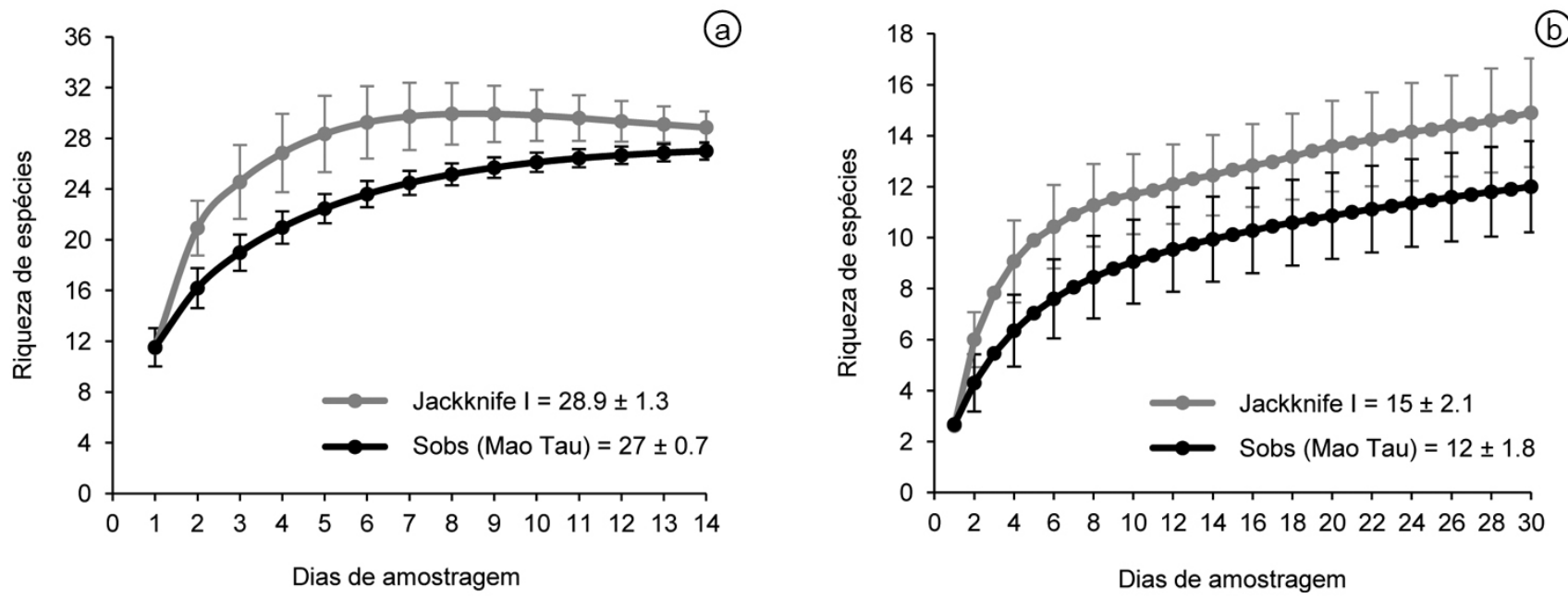

Figura 4. Curvas de rarefação representando a riqueza observada (linha preta) e estimada pelo estimador Jackknife I (linha cinza) para 14 dias de amostragem por procura auditiva e visual (a) e 30 dias por armadilhas de interceptação e queda (b) na Estação Ecológica de Santa Bárbara, SP. As barras verticais correspondem aos desvios padrão da riqueza de espécies observada e estimada.

Figure 4. Rarefaction curves representing the species richness observed (black line) and estimated by Jackknife I estimator (gray line) for 14 sampling days using acoustic and visual searches (a) and 30 days using pitfall traps (b) at the Estação Ecológica de Santa Bárbara, São Paulo state, Brazil. Vertical bars represent the standard deviations of species richness observed and estimated.

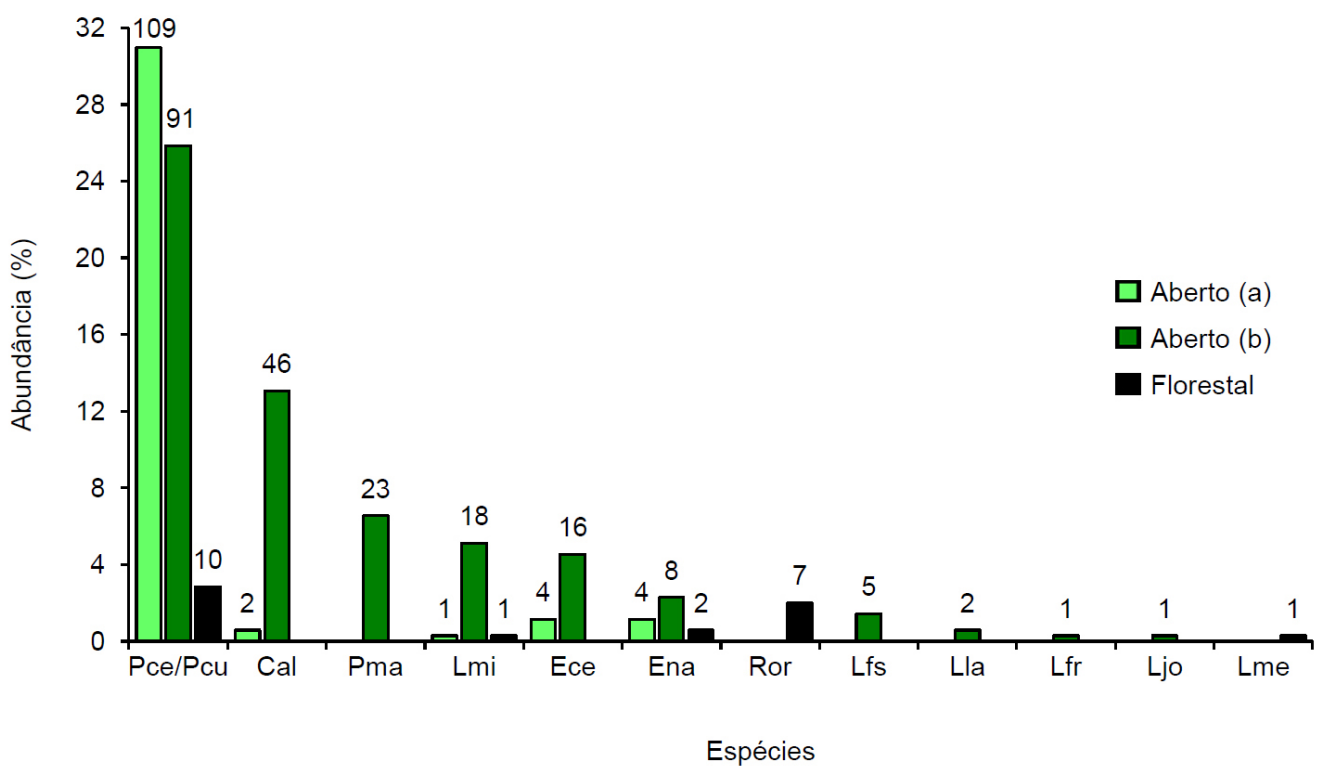

Figura 5. Abundância relativa das espécies de anuros amostradas por armadilhas de interceptação e queda nos três ambientes da Estação Ecológica de Santa Bárbara, SP. $\mathrm{Cal}=$ Chiasmocleis albopunctata $;$ Ece $=$ Elachistocleis cesarii $;$ Ena $=$ Eupemphix nattereri $; \mathrm{Lfr}=$ Leptodactylus furnarius $;$ Lfs $=$ Leptodactylus fuscus $; \mathrm{Ljo}=$ Leptodactylus jolyi $; \mathrm{Lla}=$ Leptodactylus latrans; $\mathrm{Lme}=$ Leptodactylus mystaceus $; \mathrm{Lmi}=$ Leptodactylus mystacinus; $\mathrm{Pce}=$ Physalaemus centralis; $\mathrm{Pcu}=$ Physalaemus cuvieri $;$ Pma = Physalaemus marmoratus; $\mathrm{Ror}=$ Rhinella ornata . Os valores sobre as barras representam o número de indivíduos registrados para cada espécie.

Figure 5. Relative abundance of anuran species sampled by pitfall traps in the three environments of the Estação Ecológica de Santa Bárbara, São Paulo state, Brazil. $\mathrm{Cal}=$ Chiasmocleis albopunctata $;$ Ece = Elachistocleis cesarii $;$ Ena = Eupemphix nattereri; Lfr $=$ Leptodactylus furnarius; Lfs $=$ Leptodactylus fuscus; Ljo = Leptodactylus jolyi $; \mathrm{Lla}=$ Leptodactylus latrans $;$ Lme $=$ Leptodactylus mystaceus $; \mathrm{Lmi}=$ Leptodactylus mystacinus $;$ Pce $=$ Physalaemus centralis; $\mathrm{Pcu}=$ Physalaemus cuvieri $;$ Pma $=$ Physalaemus marmoratus $;$ Ror $=$ Rhinella ornata. The values on the bars represent the number of individuals recorded for each species. 


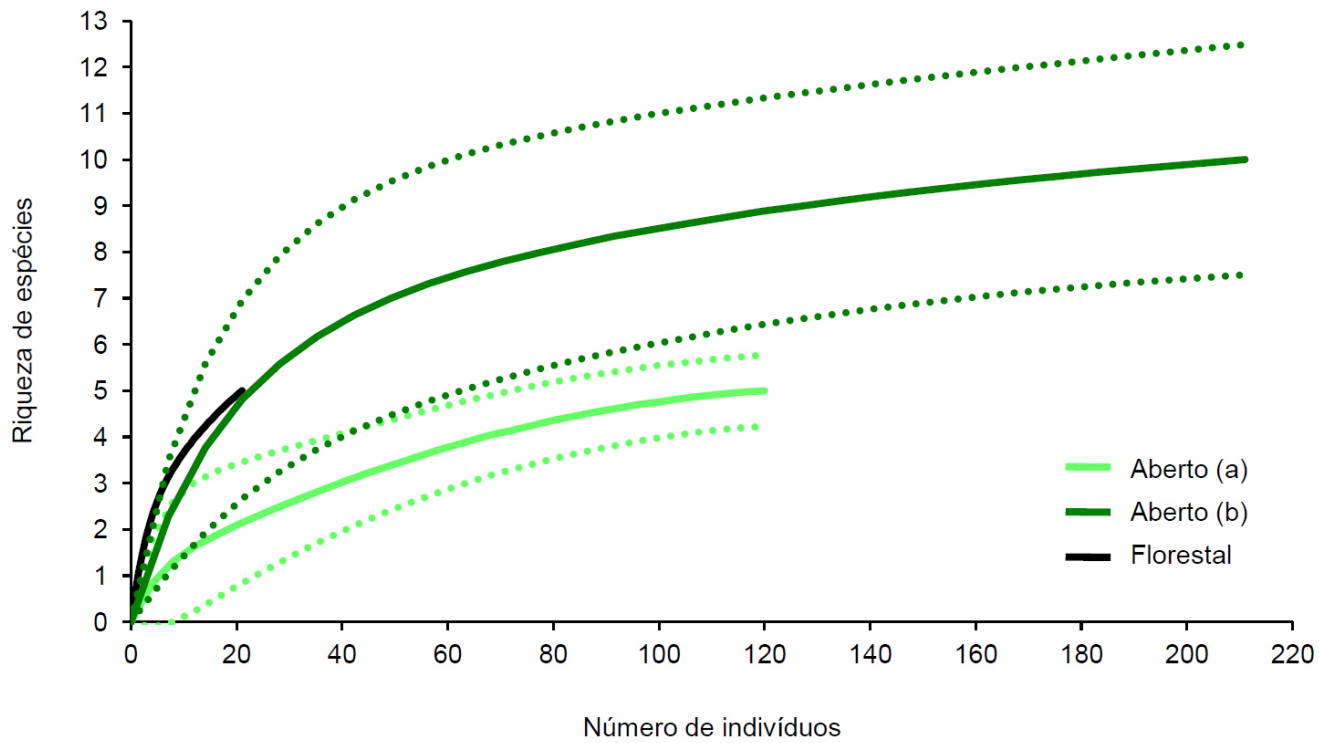

Figura 6. Curvas de rarefação dos anuros amostrados por armadilhas de interceptação e queda nos três ambientes da Estação Ecológica de Santa Bárbara, SP. As linhas pontilhadas representam os intervalos de confiança de $95 \%$.

Figure 6. Rarefaction curves of anurans sampled by pitfall traps in the three environments of the Estação Ecológica de Santa Bárbara, São Paulo state, Brazil. The dotted lines represent the confidence intervals of $95 \%$.

cinco espécies (21 indivíduos) e nos ambientes com formações abertas (a e b) foram registradas cinco espécies (120 indivíduos) e dez espécies (211 indivíduos), respectivamente (Figura 5, Figura 6). Physalaemus centralis e P. cuvieri, consideradas conjuntamente nas análises, foram as espécies mais abundantes, correspondendo a $60 \%$ do total de indivíduos amostrados (Figura 5). Considerando-se a rarefação pelo mesmo número de indivíduos capturados (21) não foi detectada diferença na riqueza de espécies entre os três ambientes (Figura 6). No entanto, foi observada diferença na riqueza de espécies quando os dois ambientes abertos foram comparados entre si para o mesmo número de indivíduos registrados (120) (Figura 6). È possível que esta diferença esteja relacionada ao fato destes ambientes abertos apresentarem históricos distintos de perturbações, sendo que um deles (aberto b) apresenta a ocorrência de eventos de fogo no passado e pastoreio de gado pouco intensivo. Adicionalmente, é provável que os ambientes abertos também possuam diferenças na disponibilidade de sítios aquáticos adequados para a reprodução das espécies.

Apesar de vários autores terem demonstrado que as espécies de anuros presentes no Cerrado apresentam uma maior associação às formações abertas deste domínio (e.g. Brandão \& Araújo 2002, Colli et al. 2002, Eterovick \& Sazima 2004, Brasileiro et al. 2005, Araujo et al. 2009, Valdujo et al. 2011), o presente estudo não detectou diferença na riqueza de espécies entre as formações abertas e florestais. No entanto, registrou-se uma maior abundância de anuros nas formações savânicas, o que reflete a maior procura dos indivíduos por corpos d'água presentes em fitofisionomias abertas durante o período reprodutivo (e.g. Brasileiro et al. 2005, Araujo et al. 2009, Araujo \& Almeida-Santos 2011, Valdujo et al. 2011). Devese ressaltar que as formações florestais são importantes ambientes mésicos, sendo utilizados por adultos e juvenis como locais de refúgio, forrageio, hibernação e migração, principalmente durante os períodos de seca (Brandão \& Araújo 2002, Silva \& Rossa-Feres 2007). Diante do exposto, nossos resultados apontam que o mosaico de formações abertas e florestais é importante para a manutenção das populações de anuros presentes na Estação Ecológica de Santa Bárbara.

\section{Agradecimentos}

Somos gratos ao gestor Adauto Fiorucci e aos funcionários da Estação Ecológica de Santa Bárbara pelo suporte logístico ao longo dos trabalhos de campo, em especial ao José Rodrigues do Prado Filho e Carlos Roberto da Silva pelo grande apoio durante a amostragem das espécies; ao Fábio Maffei e Guilherme Marson Moya pelo auxílio nas coletas efetuadas após este estudo; ao Célio Fernando Baptista Haddad e Luís Felipe Toledo pela contribuição na identificação dos espécimes e ao Instituto Florestal pelo suporte financeiro. Décio Tadeu Corrêa agradece a FAPESP, CAPES e UNESP/Assis e Selma Maria de Almeida Santos ao CNPq e a FAPESP pelo apoio financeiro.

\section{Referências Bibliográficas}

AB'SÁBER, A.N. 2005. Os domínios de natureza no Brasil: potencialidades paisagísticas. Ateliê Editorial, São Paulo.

ARAUJO, C.O. \& ALMEIDA-SANTOS, S.M. 2011. Herpetofauna de um remanescente de Cerrado no estado de São Paulo, sudeste do Brasil Biota Neotrop. 11(3): http://www.biotaneotropica.org.br/v11n3/pt/ abstract?article+bn00511032011 (último acesso em 06/06/2012).

ARAUJO, C.O. \& ALMEIDA-SANTOS, S.M. 2013. Composição, riqueza e abundância de anuros em um remanescente de Cerrado e Mata Atlântica no estado de São Paulo. Biota Neotrop. 13(1): http://www.biotaneotropica. org.br/v13n1/pt/abstract?inventory+bn02613012013 (último acesso em 06/06/2013)

ARAUJO, C.O., CONDEZ, T.H. \& SAWAYA, R.J. 2009. Anfíbios anuros do Parque Estadual das Furnas do Bom Jesus, sudeste do Brasil, e suas relações com outras taxocenoses no Brasil. Biota Neotrop. 9(2): http:// www.biotaneotropica.org.br/v9n2/pt/abstract?article+bn01109022009 (último acesso em 13/02/2010).

BRANDÃO, R.A. \& ARAÚJO, A.F.B. 2002. A herpetofauna associada a matas de galeria no Distrito Federal. In Cerrado: caracterização e recuperação de matas de galeria (J.F. Ribeiro, C.E.L. Fonseca \& J.C. Sousa-Silva, orgs.). Embrapa/CPAC, Planaltina, p.560-604.

BRASILEIRO, C.A., SAWAYA, R.J., KIEFER, M.C. \& MARTINS, M. 2005. Amphibians of an open Cerrado fragment in southeastern Brazil. Biota Neotrop. 5(2): http://www.biotaneotropica.org.br/v5n2/ pt/abstract?article+BN00405022005 (último acesso em 16/07/2010). 
BRASSALOTI, R.A., ROSSA-FERES, D.C. \& BERTOLUCI, J. 2010. Anurofauna da Floresta Estacional Semidecidual da Estação Ecológica dos Caetetus, sudeste do Brasil. Biota Neotrop. 10(1): http://www. biotaneotropica.org.br/v10n1/pt/abstract?inventory+bn01810012010 (último acesso em 16/07/2010).

CECHIN, S.Z. \& MARTINS, M. 2000. Eficiência de armadilhas de queda (Pitfall traps) em amostragens de anfíbios e répteis no Brasil. Rev. Bras. Zool. 17(3):729-749. http://dx.doi.org/10.1590/S010181752000000300017

COLLI, G.R., BASTOS, R.P. \& ARAÚJO, A.F.B. 2002. The character and dynamics of the Cerrado herpetofauna. In The cerrados of Brazil: ecology and natural history of a Neotropical Savanna (P.S. Oliveira \& R.J. Marquis, eds.). Columbia University Press, New York, p.223-241.

COLWELL, R.K. 2009. EstimateS: statistical estimation of species richness and shared species from samples. versão 8.2.0. http://viceroy.eeb.uconn. edu/estimates (último acesso em: 10/07/2010).

CORN, P.S. 1994. Straight-line drift-fences and pitfall traps. In Measuring and monitoring biological diversity. Standard methods for amphibians (W.R. Heyer, M.A. Donnely, R.W. McDiarmid, L.C. Hayek \& M.S. Foster, eds.). Smithsonian institution Press, Washington, London, p.109-117.

CORRÊA, D.T., GUIMARÃES, M., OLIVEIRA, T.A.L., MARTINS, M. \& SAWAYA, R.J. 2013. Population declines: Toe-clipping vital to amphibian research. Nature 493:304-305. PMid:23325205. http://dx.doi. org $/ 10.1038 / 493305 \mathrm{e}$

CRUMP, M.L. \& SCOTT JUNIOR, N.J. 1994. Visual encounter surveys. In Measuring and monitoring biological diversity: standard methods for amphibians (W.R. Heyer, M.A. Donnelly, R.W. McDiarmid, L.C. Hayek \& M.S Foster, eds.). Smithsonian Institution Press, Washington, London, p.84-92.

DINIZ-FILHO, J.A.F., BINI, L.M., VIEIRA, C.M., BLAMIRES, D., TERRIBILE, L., BASTOS, R., OLIVEIRA, G. \& BARRETO, B. 2008. Spatial patterns of terrestrial vertebrate species richness in the Brazilian Cerrado. Zool. Stud. 47(2):146-157.

DUELLMAN, W.E. \& TRUEB, L. 1986. Biology of amphibians. McGrawHill Publishing Company, New York. PMid:3766684.

DURIGAN, G. \& RATTER, J.A. 2006. Successional changes in Cerrado and Cerrado/forest ecotonal vegetation in Western São Paulo State, Brazil, 1962-2000. Edinburgh J. Bot. 63(1):119-130. http://dx.doi.org/10.1017/ S0960428606000357

DURIGAN, G., FRANCO, G.A.D.C. \& SIQUEIRA, M.F. 2004. A vegetação dos remanescentes de Cerrado no Estado de São Paulo. In Viabilidade da conservação dos remanescentes de Cerrado no Estado de São Paulo (M.D. Bittencourt \& R.R. Mendonça, orgs.). Annablume, FAPESP, São Paulo, p.29-56.

DURIGAN, G., PINHEIRO, E.S. \& GUERIN, N. 2011. Vegetação. In Plano de manejo da Estação Ecológica de Santa Bárbara (A.C.G., Melo \& G. Durigan, orgs.). Instituto Florestal/SEMA, São Paulo, p.73-82.

DURIGAN, G., SIQUEIRA, M.F. \& FRANCO, G.A.D.C. 2007. Threats to the Cerrado remnants of the State of São Paulo, Brazil. Braz. Sci. Agric. 64(4):355-363.

DURIGAN, G., SIQUEIRA, M.F., FRANCO, G.A.D.C., BRIDGEWATER, S. \& RATTER, J.A. 2003. The vegetation of priority areas for Cerrado conservation in São Paulo State, Brazil. Edinburgh J. Bot. 60(2):217-241. http://dx.doi.org/10.1017/S0960428603000155

EITEN, G. 1972. The cerrado vegetation of Brazil. Bot. Rev. 38:201-341. http://dx.doi.org/10.1007/BF02859158

ETEROVICK, P.C. \& SAZIMA, I. 2004. Anfíbios da Serra do Cipó, Minas Gerais - Brasil. PUC-Minas, Belo Horizonte.

FROST, D.R. 2013. Amphibian species of the world: an online reference. version 5.6. American Museum of Natural History, New York. http:// research.amnh.org/herpetology/amphibia/index.html (último acesso em 06/02/2013).
GARCIA, P.C.A., SAWAYA, R.J., MARTINS, I.A., BRASILEIRO, C.A., VERDADE, V.K., JIM, J., SEGALLA, M.V., MARTINS, M., ROSSAFERES, D.C., HADDAD, C.F.B., TOLEDO, L.F., PRADO, C.P.A., BERNECK, B.M. \& ARAÚJO, O.G.S. 2009. Anfíbios. In Fauna ameaçada de extinção no estado de São Paulo: vertebrados (M. Bressan, M.C.M. Kierulff \& A.M. Sugieda, orgs.). Fundação Parque Zoológico de São Paulo/SEMA, São Paulo, p.329-347.

GOTELLI, N.J. \& COWELL, R.K. 2001. Quantifying biodiversity: procedures and pitfalls in the measurement and comparison of species richness. Ecol. Lett. 4(4):379-391. http://dx.doi.org/10.1046/j.1461-0248.2001.00230.x

HADDAD, C.F.B., TOLEDO, L.F. \& PRADO, C.P.A. 2008. Anfíbios da Mata Atlântica: guia dos anfíbios anuros da Mata Atlântica. Neotropica, São Paulo.

HORTAL, J., BORGES, P.A.V. \& GASPARE, C. 2006. Evaluating the performance of species richness estimators: sensitivity to sample grain size. J. Anim. Ecol. 75(1):274-287. PMid:16903065. http://dx.doi. org/10.1111/j.1365-2656.2006.01048.x

INTERNATIONAL UNION FOR CONSERVATION OF NATURE - IUCN. 2013. The IUCN red list of threatened species. version 2013.1. http:// www.iucnredlist.org (último acesso em: 12/07/2013).

KLINK, C.A. \& MACHADO, R.B. 2005. Conservation of the Brazilian Cerrado. Conserv. Biol. 19(3):707-713. http://dx.doi.org/10.1111/j.15231739.2005.00702.x

KRONKA, F.J.N., NALON, M.A. \& MATSUKUMA, C.K. 2005. Inventário florestal da vegetação natural do estado de São Paulo. Instituto Florestal/ SEMA, São Paulo.

LÜDDECKE, H. \& AMÉZQUITA, A. 1999. Assessment of disc clipping on the survival and behavior of the Andean Frog Hyla labialis. Copeia 1999(3):824-830. http://dx.doi.org/10.2307/1447623

MACHADO, A.B.M., DRUMMOND, G.M. \& PAGLIA, A.P. 2008. Livro vermelho da fauna brasileira ameaçada de extinção. Fundação Biodiversitas/MMA, Belo Horizonte.

MAFFEI, F., UBAID, F.K. \& JIM, J. 2011a. Anfíbios: Fazenda Rio Claro, Lençóis Paulista, SP, Brasil. Canal 6, Bauru.

MAFFEI, F., UBAID, F.K. \& JIM, J. 2011b. Anurofauna em área de cerrado aberto no município de Borebi, estado de São Paulo, Sudeste do Brasil: uso do habitat, abundância e variação sazonal. Biota Neotrop. 11(2): http:// www.biotaneotropica.org.br/v11n2/pt/abstract?article+bn04011022011 (último acesso em 06/10/2012).

MAGURRAN, A.E. 2004. Measuring biological diversity. Blackwell Publishing Company, Oxford.

MELO, A.C.G. \& DURIGAN, G. 2011. Plano de manejo da Estação Ecológica de Santa Bárbara. Instituto Florestal/SEMA, São Paulo.

MELO, A.S. \& FROEHLICH, C.G. 2001. Evaluation of methods for estimating macroinvertebrate species richness using individual stones in tropical streams. Freshwater Biol. 46:711-721. http://dx.doi.org/10.1046/ j.1365-2427.2001.00709.x

MELO, A.S. 2004. A critique of the use of jackknife and related non-parametric techniques to estimate species richness. Community Ecol. 5(2):149-157. http://dx.doi.org/10.1556/ComEc.5.2004.2.1

MITTERMEIER, R.A., GIL, P.R., HOFFMAN, M., PILGRIM, J., BROOKS, T., MITTERMEIER, C.G., LAMOREUX, J. \& FONSECA, G.A.B. 2004. Hotspots revisited: earth's biologically richest and most endangered terrestrial ecoregions. CEMEX \& Agrupacion Sierra Madre, Cidade do México.

MYERS, N., MITTERMEIER, R.A., MITTERMEIER, C.G., FONSECA, G.A.B. \& KENT, J. 2000. Biodiversity hotspots for conservation priorities. Nature 403:853-858. PMid:10706275. http://dx.doi. org/10.1038/35002501

OLIVEIRA-FILHO, A.T. \& RATTER, J.A. 2002. Vegetation physiognomies and woody flora of the Cerrado biome. In The cerrados of Brazil: ecology and natural history of a Neotropical Savanna (P.S. Oliveira \& R.J. Marquis, orgs.). Columbia University Press, NewYork, p.91-120. 
PEEL, M.C., FINLAYSON, B.L. \& McMAHON, T.A. 2007. Updated world map of the Köppen-Geiger climate classification. Hydrol. Earth Syst. Sci. 11:1633-1644. http://dx.doi.org/10.5194/hess-11-1633-2007

PYRON, R.A. \& WIENS, J.J. 2011. A large-scale phylogeny of Amphibia including over 2800 species, and a revised classification of extant frogs, salamanders, and caecilians. Mol. Phylogenet. Evol. 61(2):543-583. PMid:21723399. http://dx.doi.org/10.1016/j.ympev.2011.06.012

PHILLOTT, A.D., SKERRATT, L.F., McDONALD, K.R., LEMCKERT, F.L., HINES, H.B., CLARKE, J.M., ALFORD, R.A. \& SPEARE, R. 2007. Toe-clipping as an acceptable method of identifying individual anurans in mark recapture studies. Herpetol. Rev. 38(3):305-308.

PIVELLO, V.R., CARVALHO, V.M.C., LOPES, P.F., PECCININI, A.A \& ROSSO, S. 1999. Abundance and distribution of native and alien grasses in a Cerrado (Brazilian savanna) biological reserve. Biotropica 31(1):71-82.

POUGH, F.H., ANDREWS, R.M., CADLE, J.E., CRUMP, M.L., SAVITZKY, A.H. \& WELLS, K.D. 2004. Herpetology. Pearson Prentice-Hall, New Jersey.

RIBEIRO, J.F. \& WALTER, B.M.T. 2008. As principais fitofisionomias do Bioma Cerrado. In Cerrado: ecologia e flora (S.M. Sano, S.P. Almeida \& J.F. Ribeiro, eds.). Embrapa, Planaltina, p.153-212.

RIBEIRO-JÚNIOR, J.W. \& BERTOLUCI, J. 2009. Anuros do cerrado da Estação Ecológica e da Floresta Estadual de Assis, sudeste do Brasil. Biota Neotrop. 9(1): http://www.biotaneotropica.org.br/v9n1/pt/abstrac t?inventory+bn02709012009 (último acesso em 16/07/2011).

ROSSA-FERES, D.C., MARTINS, M., MARQUES, O.A.V., MARTINS, I.A., SAWAYA, R.J. \& HADDAD, C.F.B. 2008. Herpetofauna. In Diretrizes para a conservação e restauração da biodiversidade no estado de São Paulo (R.R. Rodrigues \& V.LR. Bononi, orgs.). Instituto de Botânica/ SEMA, São Paulo, p.82-94.

ROSSA-FERES, D.C., SAWAYA, R.J., FAIVOVICH, J., GIOVANELLI, J.G.R., BRASILEIRO, C.A., SCHIESARI, L., ALEXANDRINO, J. \& HADDAD, C.F.B. 2011. Anfíbios do Estado de São Paulo, Brasil: Conhecimento Atual e Perspectivas. Biota Neotrop. 11(1a): http://www. biotaneotropica.org.br/v11n1a/pt/abstract?inventory+bn0041101a2011 (último acesso em 22/02/2012).

SANO, E.E., ROSA, R., BRITO, J.L.S. \& FERREIRA, L.G. 2010. Land cover mapping of the tropical savanna region in Brazil. Environ. Monit. Assess. 166:113-124. PMid:19504057. http://dx.doi.org/10.1007/s10661009-0988-4

SANTOS, T.G., VASCONCELOS, T.S., ROSSA-FERES, D.C. \& HADDAD, C.F.B. 2009. Anurans of a seasonally dry tropical forest: Morro do Diabo State Park, São Paulo state, Brazil. J. Nat. Hist. 43(15-16):973-993. http:// dx.doi.org/10.1080/00222930802702498

SCOTT JUNIOR., N.J., WOODWARD, B.D. 1994. Surveys at breeding sites In Measuring and monitoring biological diversity: standard methods for amphibians (W.R. Heyer, M.A. Donnelly, R.W. McDiarmid, L.C. Hayek \& M.S Foster, eds.). Smithsonian Institution Press. Washington and London, p.118-125.
SILVA, F.R. 2010. Evaluation of survey methods for sampling anuran species richness in the Neotropics. SAJH 5(3):212-220. http://dx.doi. org/10.2994/057.005.0307

SILVA, F.R. \& ROSSA-FERES, D.C. 2007. Uso de fragmentos florestais por anuros (Amphibia) de área aberta na região noroeste do Estado de São Paulo. Biota Neotrop. 7(2): http://www.biotaneotropica.org.br/v7n2/pt/ abstract?article+bn03707022007 (último acesso em 06/06/2012).

SILVA, J.M.C. \& BATES, J.M. 2002. Biogeographic patterns and conservation in the South American Cerrado: a tropical savanna hotspot. BioScience 52(3):225-233. http://dx.doi.org/10.1641/0006-3568(2002)052[0225:BP ACIT]2.0.CO;2

SILVANO, D. 2011. Distribuição e conservação de anfíbios no Cerrado em cenários atuais e futuros. Tese de Doutorado, Universidade de Brasília, Brasília.

SILVEIRA, L.F., BEISIEGEL, B.M., CURCIO, F.F., VALDUJO, P.H., DIXO, M., VERDADE, V.K., MATTOX, G.M.T. \& CUNNINGHAM, P.T.M. 2010. Para que servem os inventários de fauna? Estudos avançados 68:173-207. http://dx.doi.org/10.1590/S0103-40142010000100015

TOLEDO, L.F., GIOVANELLI, J.G.R., GIASSON, L.O.M., PRADO, C.P.A., GUIMARÃES, L.D., BASTOS, R.P. \& HADDAD, C.F.B. 2007. Guia interativo dos anfíbios anuros do Cerrado, Campos Rupestres e Pantanal. Neotrópica, São Paulo. CD-ROM.

UETANABARO, M., PRADO, C.P.A., RODRIGUES, D.J., GORDO, M. \& CAMPOS, Z. 2008. Guia de campo dos anuros do Pantanal e planaltos de entorno. UFMS, Campo Grande, UFMT, Cuiabá. PMid:17713858.

VALDUJO, P.H. 2011. Diversidade e distribuição de anfíbios no Cerrado: o papel dos fatores históricos e dos gradientes ambientais. Tese de Doutorado, Universidade de São Paulo, São Paulo.

VALDUJO, P.H., CAMACHO, A., RECODER, R.S., TEIXEIRA JUNIOR, M., GHELLERE, J.M.B., MOTT, T., NUNES, P.M.S., NOGUEIRA, C. \& RODRIGUES, M.T. 2011. Anfíbios da Estação Ecológica Serra Geral do Tocantins, região do Jalapão, Estados do Tocantins e Bahia. Biota Neotrop. 11(1): http://www.biotaneotropica.org.br/v11n1/pt/ abstract?article+bn03511012011. (último acesso em 22/07/2012).

VALDUJO, P.H., SILVANO, D.L., COLLI, G. \& MARTINS, M. 2012. Anuran species composition and distribution patterns in Brazilian Cerrado, a neotropical hotspot. S. A. J. H. 7(2):63-78. http://dx.doi. org/10.2994/057.007.0209

VERDADE, V.K., VALDUJO, P.H., CARNAVAL, A.C., SCHIESARI, L., TOLEDO, L.F., MOTT, T., ANDRADE, G.V., ETEROVICK, P.C., MENIN, M., PIMENTA, B.V.S., NOGUEIRA, C., LISBOA, C.S., PAULA, C.D. \& SILVANO, D.L. 2012. A leap further: the Brazilian Amphibian Conservation Action Plan. Alytes 29(1-4):28-43.

WALTHER, B.A. \& MOORE, J.L. 2005. The concepts of bias, precision and accuracy, and their use in testing the performance of species richness estimators, with a literature review of estimator performance. Ecography 28(6):815-829. http://dx.doi.org/10.1111/j.2005.0906-7590.04112.x 
Araujo, C.O. et al.

Apêndice 1. Linhas de armadilhas de interceptação e queda e ambientes aquáticos amostrados na Estação Ecológica de Santa Bárbara, SP.

Appendix 1. Pitfall traps lines and aquatic sites sampled at the Estação Ecológica de Santa Bárbara, São Paulo state, Brazil.

\begin{tabular}{|c|c|c|c|}
\hline $\begin{array}{l}\text { Linha de armadilha/ } \\
\text { ambiente aquático }\end{array}$ & Fitofisionomia & $\begin{array}{c}\text { Tipo de } \\
\text { ambiente }\end{array}$ & $\begin{array}{c}\text { Coordenada geográfica } \\
\text { (SAD 69) }\end{array}$ \\
\hline 1 & cerradão & florestal & $22^{\circ} 50^{\prime} 11^{\prime \prime} \mathrm{S}$ e $49^{\circ} 13^{\prime} 56^{\prime \prime} \mathrm{O}$ \\
\hline 2 & cerradão & florestal & $22^{\circ} 50^{\prime} 13^{\prime \prime} \mathrm{S}$ e $49^{\circ} 14^{\prime} 04^{\prime \prime} \mathrm{O}$ \\
\hline 3 & mata seca semidecídua & florestal & $22^{\circ} 50^{\prime} 04^{\prime \prime} \mathrm{S}$ e $49^{\circ} 14^{\prime} 15^{\prime \prime} \mathrm{O}$ \\
\hline 4 & cerradão & florestal & $22^{\circ} 49^{\prime} 37^{\prime \prime} \mathrm{S}$ e $49^{\circ} 13^{\prime} 45^{\prime \prime} \mathrm{O}$ \\
\hline 5 & cerradão & florestal & $22^{\circ} 49^{\prime} 52^{\prime \prime} \mathrm{S}$ e $49^{\circ} 13^{\prime} 44^{\prime \prime} \mathrm{O}$ \\
\hline 6 & cerradão & florestal & $22^{\circ} 49^{\prime} 31^{\prime \prime} \mathrm{S}$ e $49^{\circ} 14^{\prime} 21^{\prime \prime} \mathrm{O}$ \\
\hline 7 & cerrado típico & aberto (a) & $22^{\circ} 48^{\prime} 42^{\prime \prime} \mathrm{S}$ e $49^{\circ} 11^{\prime} 57^{\prime \prime} \mathrm{O}$ \\
\hline 8 & cerrado típico & aberto (a) & $22^{\circ} 48^{\prime} 50^{\prime \prime} \mathrm{S}$ e $49^{\circ} 12^{\prime} 09^{\prime \prime} \mathrm{O}$ \\
\hline 9 & cerrado típico & aberto (a) & $22^{\circ} 48^{\prime} 51^{\prime \prime} \mathrm{S}$ e $49^{\circ} 12^{\prime} 30^{\prime \prime} \mathrm{O}$ \\
\hline 10 & cerrado típico/cerrado ralo & aberto (a) & $22^{\circ} 48^{\prime} 13^{\prime \prime} \mathrm{S}$ e $49^{\circ} 11^{\prime} 30^{\prime \prime} \mathrm{O}$ \\
\hline 11 & cerrado típico & aberto (a) & $22^{\circ} 48^{\prime} 18^{\prime \prime} \mathrm{S}$ e $49^{\circ} 11^{\prime} 14^{\prime \prime} \mathrm{O}$ \\
\hline 12 & cerrado típico & aberto (a) & $22^{\circ} 48^{\prime} 23^{\prime \prime} \mathrm{S}$ e $49^{\circ} 10^{\prime} 59^{\prime \prime} \mathrm{O}$ \\
\hline 13 & cerrado ralo & aberto (b) & $22^{\circ} 47^{\prime} 06^{\prime \prime} \mathrm{S}$ e $49^{\circ} 14^{\prime} 29^{\prime \prime} \mathrm{O}$ \\
\hline 14 & cerrado ralo & aberto (b) & $22^{\circ} 47^{\prime} 13^{\prime \prime} \mathrm{S}$ e $49^{\circ} 14^{\prime} 05^{\prime \prime} \mathrm{O}$ \\
\hline 15 & cerrado típico & aberto (b) & $22^{\circ} 47^{\prime} 24^{\prime \prime} \mathrm{S}$ e $49^{\circ} 14^{\prime} 37^{\prime \prime} \mathrm{O}$ \\
\hline 16 & cerrado típico & aberto (b) & $22^{\circ} 47^{\prime} 30^{\prime \prime} \mathrm{S}$ e $49^{\circ} 15^{\prime} 07^{\prime \prime} \mathrm{O}$ \\
\hline 17 & cerrado típico & aberto (b) & $22^{\circ} 47^{\prime} 13^{\prime \prime} \mathrm{S}$ e $49^{\circ} 14^{\prime} 59^{\prime \prime} \mathrm{O}$ \\
\hline 18 & cerrado típico & aberto (b) & $22^{\circ} 46^{\prime} 56^{\prime \prime} \mathrm{S}$ e $49^{\circ} 14^{\prime} 56^{\prime \prime} \mathrm{O}$ \\
\hline I - lago & cerradão alterado & aberto & $22^{\circ} 49^{\prime} 39^{\prime \prime} \mathrm{S}$ e $49^{\circ} 14^{\prime} 10^{\prime \prime} \mathrm{O}$ \\
\hline II - brejo & campo limpo úmido & aberto & $22^{\circ} 49^{\prime} 30^{\prime \prime} \mathrm{S}$ e $49^{\circ} 14^{\prime} 51^{\prime \prime} \mathrm{O}$ \\
\hline III - brejo & campo limpo úmido & aberto & $22^{\circ} 49^{\prime} 17^{\prime \prime} \mathrm{S}$ e $49^{\circ} 14^{\prime} 53^{\prime \prime} \mathrm{O}$ \\
\hline IV - riacho & ecótono Cerrado/mata seca semidecídua & florestal & $22^{\circ} 48^{\prime} 57^{\prime \prime} \mathrm{S}$ e $49^{\circ} 14^{\prime} 27^{\prime \prime} \mathrm{O}$ \\
\hline V - lago & ecótono Cerrado/mata seca semidecídua & florestal & $22^{\circ} 48^{\prime} 55^{\prime \prime} \mathrm{S}$ e $49^{\circ} 14^{\prime} 26^{\prime \prime} \mathrm{O}$ \\
\hline VI - lagoa & mata de galeria alterada & aberto & $22^{\circ} 48^{\prime} 49^{\prime \prime} \mathrm{S}$ e $49^{\circ} 14^{\prime} 10^{\prime \prime} \mathrm{O}$ \\
\hline VII - charco & mata de galeria alterada & aberto & $22^{\circ} 48^{\prime} 25^{\prime \prime} \mathrm{S}$ e $49^{\circ} 12^{\prime} 39^{\prime \prime} \mathrm{O}$ \\
\hline VIII - brejo/riacho & campo limpo úmido/mata de galeria & aberto/fechado & $22^{\circ} 48^{\prime} 27^{\prime \prime} \mathrm{S}$ e $49^{\circ} 12^{\prime} 38^{\prime \prime} \mathrm{O}$ \\
\hline IX - brejo & campo limpo úmido & aberto & $22^{\circ} 48^{\prime} 07^{\prime \prime} \mathrm{S}$ e $49^{\circ} 11^{\prime} 49^{\prime \prime} \mathrm{O}$ \\
\hline $\mathrm{X}$ - brejo & campo limpo úmido & aberto & $22^{\circ} 48^{\prime} 22^{\prime \prime} \mathrm{S}$ e $49^{\circ} 10^{\prime} 56^{\prime \prime} \mathrm{O}$ \\
\hline XI - poça temporária & reflorestamento de Pinus sp. & florestal & $22^{\circ} 47^{\prime} 43^{\prime \prime} \mathrm{S}$ e $49^{\circ} 13^{\prime} 22^{\prime \prime} \mathrm{O}$ \\
\hline XII - riacho & mata de galeria & florestal & $22^{\circ} 47^{\prime} 42^{\prime \prime} \mathrm{S}$ e $49^{\circ} 14^{\prime} 09^{\prime \prime} \mathrm{O}$ \\
\hline XIII - brejo & campo limpo úmido & aberto & $22^{\circ} 47^{\prime} 19^{\prime \prime} \mathrm{S}$ e $49^{\circ} 14^{\prime} 35^{\prime \prime} \mathrm{O}$ \\
\hline XIV - brejo & campo limpo úmido & aberto & $22^{\circ} 47^{\prime} 25^{\prime \prime} \mathrm{S}$ e $49^{\circ} 15^{\prime} 21^{\prime \prime} \mathrm{O}$ \\
\hline
\end{tabular}

Apêndice 2. Anuros coletados na Estação Ecológica de Santa Bárbara, SP e depositados na Coleção de Anfíbios do Instituto de Biociências da Universidade Estadual Paulista, Rio Claro (CFBH) e Coleção Herpetológica do Museu de Zoologia da Universidade de São Paulo, São Paulo (MZUSP).

Appendix 2. Anuran species collected at the Estação Ecológica de Santa Bárbara, São Paulo state, Brazil and deposited in the Coleção de Anfíbios do Instituto de Biociências da Universidade Estadual Paulista, Rio Claro (CFBH) and Coleção Herpetológica do Museu de Zoologia da Universidade de São Paulo, São Paulo (MZUSP).

CFBH: Scinax berthae (22335-42).

MZUSP: Aplastodiscus perviridis (151012); Chiasmocleis albopunctata (151024-28); Dendropsophus elianeae (151058-61); Dendropsophus jimi (151018-23); Dendropsophus minutus (151056-57); Dendropsophus nanus (151098-102); Elachistocleis bicolor (151013); Elachistocleis cesarii (151041-49); Eupemphix nattereri (151070-73); Hypsiboas albopunctatus (151031-32); Hypsiboas caingua (151010-11); Hypsiboas lundii (151077-78); Itapotihyla langsdorffi (151063); Leptodactylus furnarius (151088-89); Leptodactylus fuscus (151004-09); Leptodactylus jolyi (151034-35); Leptodactylus labyrinthicus (151096); Leptodactylus latrans (151039-40); Leptodactylus mystaceus (151033); Leptodactylus mystacinus (151066-69); Phyllomedusa tetraploidea (151074-76); Physalaemus centralis (151079-82); Physalaemus cuvieri (151014-17); Physalaemus marmoratus (151062-65); Pseudopaludicola cf. murundu (151097); Rhinella ornata (151036-37); Rhinella schneideri (151038); Scinax fuscomarginatus (151050-55); Scinax fuscovarius (151029-30); Scinax similis (151090-95); Scinax squalirostris (151084-87). 